\title{
Variations
}

Variations

Revue internationale de théorie critique

$17 \mid 2012$

Critique du travail

\section{Travail forcé et ethos du travail}

\section{Claus Peter Ortlieb}

Traducteur : Sînziana

\section{OpenEdition}

Journals

Édition électronique

URL : http://journals.openedition.org/variations/357

DOI : 10.4000/variations.357

ISSN : 1968-3960

Éditeur

Les amis de Variations

Référence électronique

Claus Peter Ortlieb, «Travail forcé et ethos du travail », Variations [En ligne], 17 | 2012, mis en ligne le 15 octobre 2012, consulté le 19 avril 2019. URL : http://journals.openedition.org/variations/357 ; DOI : 10.4000/variations.357

Ce document a été généré automatiquement le 19 avril 2019.

Les ami•e•s de Variations 


\title{
Travail forcé et ethos du travail
}

\author{
Claus Peter Ortlieb
}

Traduction : Sînziana

\section{NOTE DE L'AUTEUR}

Version augmentée du texte publié dans Konkret $\mathrm{n}^{\circ} 5$, 2012, http://www.exit-online.org/ textanz1.php?tabelle=autoren\&index=3\&posnr=506\&backtext1=text1.php

Les méthodes de production modernes ont rendu possibles le confort et la sécurité pour tous ; à la place, nous avons choisi le surmenage pour les uns et la famine pour les autres. Jusqu'à présent nous avons continué à déployer la même activité qu'au temps où il n'y avait pas de machines; en cela nous nous sommes montrés stupides, mais rien ne nous oblige à persévérer éternellement dans cette stupidité.

Bertrand Russell, Eloge de l'oisiveté, 1932

Quatre-vingt ans et une crise économique mondiale plus tard, notre intelligence n'a manifestement guère progressé, au contraire : si depuis lors la productivité du travail dans l'industrie et l'agriculture s'est vue grosso modo décuplée, on ne peut pas dire qu'elle ait apporté à tous confort et sécurité. L'Europe, qui certes, pour le moment, s'est sort encore relativement bien, assiste à une hausse record de son taux de chômage. Quant aux quelques îlots qui demeurent compétitifs au plan global, ils luttent depuis des années déjà contre les nouvelles pandémies provoquées par la contraction progressive de l'offre de travail : du burn-out-syndrom ${ }^{1}$ à la mort subite due au surmenage en passant par la consommation routinière de produits psychopharmaceutiques.

Gardons-nous cependant d'imaginer que cette ardeur excessive au travail constatée par Russell ne serait rien d'autre qu'une habitude devenue obsolète et qu'il nous suffirait de laisser tomber - une habitude héritée du temps où il n'y avait pas de machines. Au Moyen Age, où le travail comme fin en soi était chose inconnue, on travaillait en fait moins qu'aujourd'hui. La raison en est simple : le travail tel que nous l'entendons, c'est-à-dire la dépense abstraite d'énergie humaine indépendamment de tout contenu particulier, est historiquement spécifique. On ne le rencontre que sous le capitalisme. Dans n'importe 
quelle autre formation sociale, l'idée aujourd'hui si universellement répandue selon laquelle « un travail, quel qu'il soit, vaut mieux que pas de travail » aurait paru, à juste titre, complètement délirante.

3 Ce délire est le principe abstrait qui régit les rapports sociaux sous le capitalisme. Si l'on fait abstraction des activités criminelles, le travail - qu'il s'agisse du nôtre ou de l'appropriation de celui d'autrui - est pour nous l'unique moyen de participer à la société. Mais, en même temps, il ne dépend pas du contenu de l'activité en question; que je fasse pousser des pommes de terre ou que je fabrique des bombes à fragmentation n'a aucune importance, du moment que mon produit trouve un acheteur et transforme ainsi mon argent en davantage d'argent. Base de la valorisation de la valeur, le travail constitue une fin en soi et un principe social contraignant dont l'unique but consiste à accumuler toujours plus de « travail mort » sous forme de capital.

4 Une contrainte à laquelle tout est soumis dans la même mesure ne se maintiendra durablement qu'à condition que ceux qu'elle ligote apprennent à aimer leurs chaînes. En cela aussi la société bourgeoise se distingue des précédentes. D'Aristote à Thomas d'Aquin en passant par Augustin, les philosophes de l'Antiquité et du Moyen Age ont célébré l'oisiveté - et surtout pas le travail - comme la voie menant à une vie heureuse ${ }^{2}$ :

Au dire de la plupart des hommes, le bonheur ne va pas sans le plaisir.

Aristote (384 - 322 av. J.C.), Ethique à Nicomaque

L'apprentissage de la vertu est incompatible avec une vie d'artisan et de manœuvre.

Aristote, Politique

Quittons ces vaines et creuses occupations : abandonnons tout le reste pour la recherche de la vérité.

Augustin (354 - 430 ap. J.C.), Les Confessions

Absolument et de soi la vie contemplative est plus parfaite que la vie active.

Thomas d'Aquin (1125 - 1274), Somme théologique

D'autres ne seront pas du même avis, tels par exemple les fondateurs de certains ordres monastiques qui verront dans le travail un moyen d'atteindre l'ascèse et l'abstinence. Mais c'est seulement au protestantisme qu'il reviendra d'en faire un principe à grande échelle, appliqué à l'ensemble de la population :

L'oisiveté est péché contre le commandement de Dieu, car Il a ordonné qu'ici-bas chacun travaille.

Martin Luther $(1483$ - 1546)

Et les Lumières n'auront de cesse d'élever l'ethos du travail, autrement dit l'obligation morale de travailler, au rang de fin en soi :

Il est de la plus haute importance que les enfants apprennent à travailler. L'homme est le seul animal qui doit travailler.

Kant, Réflexions sur l'éducation, 1803

La plus grande perfection morale possible de l'homme est de remplir son devoir et par devoir.

Kant, Principes métaphysiques de la morale, 1797

Il n'existe qu'une seule échappatoire au travail : faire travailler les autres pour soi. 
Kant, Critique du jugement, 1790

De ces trois vices : la paresse, la lâcheté, la fausseté, le premier semble être le plus méprisable.

Kant, Anthropologie d'un point de vue pragmatique, 1798

Que l'on s'informe tout particulièrement sur les personnes qui se distinguent par une conduite indigne ! On découvrira invariablement soit qu'elles n'ont pas appris à travailler, soient qu'elles fuient le travail.

Fichte, Discours à la nation allemande, 1807

7 étroitement lié à la haine des oisifs :

Chacun doit pouvoir vivre de son travail, dit un principe avancé. Ce pouvoir-vivre est donc conditionné par le travail et n'existe nullement là où la condition ne serait pas remplie.

\section{Fichte, Fondement du droit naturel, 1796}

Dans les pays chauds, l'homme est mûr plus tôt à tous égards mais n'atteint pas la perfection des zones tempérées. L'humanité dans sa plus grande perfection se trouve dans la race blanche. Les Indiens jaunes n'ont que peu de capacités, les Noirs leur sont bien inférieurs encore, et au plus bas de l'échelle se placent certaines peuplades américaines.

\section{Kant, Géographie physique, 1802}

Le barbare est paresseux et se distingue de l'homme civilisé en ceci qu'il reste plongé dans son abrutissement, car la formation pratique consiste précisément dans l'habitude et dans le besoin d'agir.

Hegel, Principes de la philosophie du droit, 1820

8 Ces propos excluants et racistes sous la plume des philosophes des Lumières ne sont nullement de simples accidents de parcours mais relèvent au contraire de l'essence même de l'idéologie du travail. Parce que ce courant de pensée transfigure le travail en véritable but de l'existence de «l'homme », tous les désœuvrés se voient par contrecoup exclus de la « race humaine » : l'homme est tenu de travailler; partant, celui qui ne travaille pas ne peut prétendre au statut d'être humain à part entière.

Ce qui s'exprime ici, c'est la colère du bourreau de travail blanc envers la pression qu'il s'est lui-même imposée, une colère qui prend pour cible tout ce qui fait mine de ne pas se soumettre à ladite pression et de mener une existence oisive : les femmes, en charge de la « vraie vie » au sein de la sphère privée - dissociée du travail - de la famille bourgeoise ; toutes sortes de peuples (les attributions sont, cette fois, plus variées) vivant, sans travailler, d'amour et d'eau fraîche; ou encore le "capital accapareur ${ }^{3}$ ", qui s'approprie sans travailler la survaleur créée par d'autres. Les idéologies modernes du sexisme, du racisme, de l'antitsiganisme et de l'antisémitisme sont fondées, elles aussi, sur l'ethos du travail.

10 À partir des années 1970, en faisant disparaitre du procès de production des quantités toujours croissantes de travail, le potentiel de rationalisation de la microélectronique a plongé le capitalisme dans la crise. Pour autant, la pression intérieure et extérieure qui pousse les hommes à travailler n'a pas diminué mais s'est même au contraire accentuée à mesure que se raréfiaient les «emplois ». Pour les laissés pour compte, les conditions se 
sont durcies : ils sont désormais trop nombreux pour que leur entretien humain reste longtemps encore compatible avec le maintien de la compétitivité au plan global. La "nécessité incontournable de ramener les hommes au travail» (Angela Merkel) ne fait qu'obscurcir la perception du problème: la responsabilité du chômage ne serait plus imputable à la disparition progressive du travail mais aux chômeurs eux-mêmes, qu'il faudrait par conséquent ramener, par tous les moyens de coercition dont on dispose, à un travail qui n'existe plus. Quelque chose de semblable se déroule également au niveau européen : on impose aux " pays en faillite " restés à la traîne de l'Europe des politiques d'austérité grâce auxquelles ils sont censés, une fois cette pénible épreuve traversée, redevenir compétitifs. C'est aussi crédible que si la Fédération allemande de football prétendait, par un entraînement approprié, hisser tous à la fois les dix-huit clubs de la Bundesliga $^{4}$ aux quatre places possibles en Ligue des champions ${ }^{5}$.

Il n'y a manifestement d'issue que dans l'abolition du travail, mais cela implique bien sûr d'abolir également le capitalisme. S'y oppose en outre notre ethos du travail, fruit de plusieurs siècles de dressage :

D'aucuns diront qu'il est certes agréable d'avoir un peu de loisir, mais que les gens ne sauraient pas comment remplir leurs journées s'ils n'avaient à travailler que quatre heures par jour. Dans la mesure où cela est vrai dans le monde moderne, cela constitue un reproche adressé à notre civilisation ; à toute autre époque antérieure, ce n'aurait pas été le cas.

Bertrand Russell, Eloge de l'oisiveté, 1932

12 Le sort que Hegel assignait aux «barbares » nous revient donc : celui qui est sans emploi n'a plus qu'à rester "plongé dans son abrutissement». Autrement dit: si le sujet bourgeois répugne tellement à imaginer sa vie sans le travail, c'est aussi parce que derrière son ethos du travail rôde la peur panique de sa propre vacuité.

\section{NOTES}

1. NDT: Syndrome d'épuisement professionnel.

2. On trouvera cette citation et presque toutes les suivantes sur le très intéressant site internet www.otium-bremen.de

3. NDT: Allusion à la vision nazie (mais qui est aussi celle d'une partie de la gauche) opposant un bon capital créateur (schaffende Kapital) à un mauvais capital accapareur (raffende Kapital).

4. NDT: Le championnat fédéral allemand.

5. NDT: Le championnat européen. 
INDEX

Mots-clés : critique du travail, correspondance, artistes, socialisme vert, travailleurs culturels, Wertkritik, Gorz André, dialogue, Vincent Jean-Marie, écologie, modèles alternatifs, Marx Karl, penser le Capital, ethos du travail, Eglise Orthodoxe, salariat, domination

\section{AUTEURS}

\section{CLAUS PETER ORTLIEB}

Mathématicien et membre de la revue allemande Exit - Crise et critique de la société marchande ( http://www.exit-online.org/). 